\title{
Concept of a GaN-LED-based positioning system using structured illumination
}

\author{
Johannes Herrnsdorf, Michael J. Strain, Erdan Gu, and Martin D. Dawson \\ Institute of Photonics, University of Strathclyde, Glasgow G1 1RD (UK), Email: johannes.herrnsdorf@strath.ac.uk
}

\begin{abstract}
Accurate self-orientation within a space can be achieved using only a simple photodetector and a remote Gallium Nitride micro-light-emitting diode array, emitting a time series of varying spatial illumination patterns onto the scene.
\end{abstract}

\section{INTRODUCTION}

With the advent of light-emitting diode (LED) based lighting, much excitement has centered around so-called "smart lighting", which is energy saving on the one hand but on the other offers advanced functionality. This functionality may include intelligent dimming, active color balance, electronic control systems and data transmission. These schemes are enabled by favorable properties of LED-based lighting systems such as low power consumption, compact dimensions, easy integration with control electronics and color converters, availability of LEDs throughout the visible spectrum and fast modulation response.

Here, we present a scheme of smart lighting that allows accurate determination of the position of devices within the illuminated area. The LED ceiling light is comprised of an array of microscopic LED elements which display a pattern sequence. Devices within the illumination area monitor the pattern sequence and thus determine their position. An uplink from the device to the pattern generator is not needed. Such a system can in principle be part of a multi-user visible light communications Li-Fi (light fidelity) system [1].

We present preliminary experimental results that demonstrate the principle. In this case the position of an optical fibre tip within a certain area is tracked.

\section{Positioning With Structured Illumination}

A schematic of the system is shown in figure 1. The ceiling lighting is comprised of an LED array and optics which image the array onto the floor level. Thus, any device equipped with a photodetector at floor level detects the emission of one corresponding pixel within the array. In the simplest case, positioning can now be achieved by raster-scanning through the array, i.e. one pixel is switched on at a time. Therefore, the device can identify from which pixel it receives a signal and thus knows its position. This requires synchronization of source and detector which can be achieved by a clock signal.

A more practical implementation employs a sequence of suitable patterns rather than raster scanning. In this work, we employ the so called Hadamard basis which has been used for image compression and single pixel cameras [2]. Two examples of these illumination patterns are shown in figure $2 b$ ) and c). All patterns in this basis set have exactly half the pixels

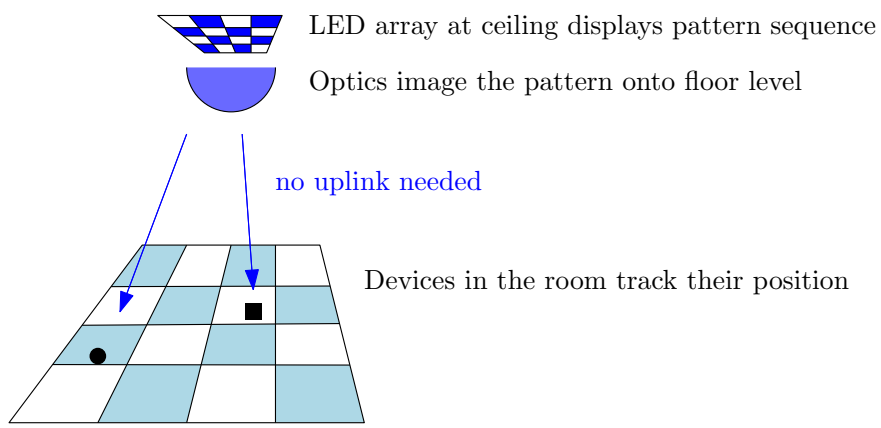

Fig. 1. Schematic of the positioning system.

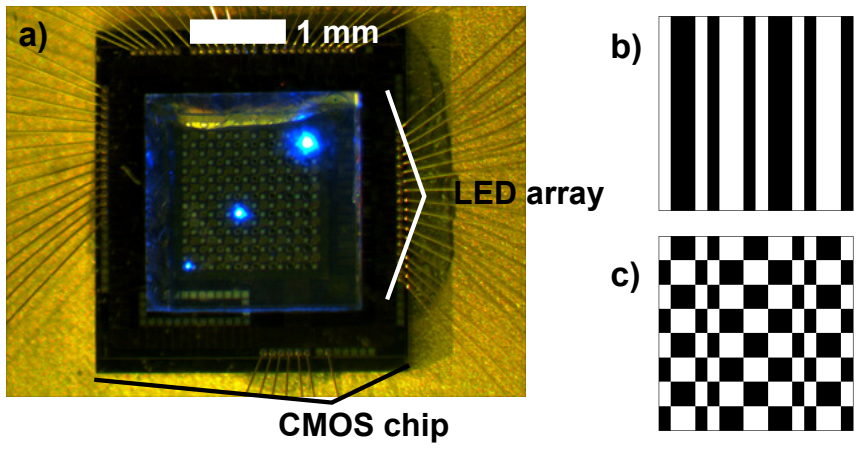

Fig. 2. a) Micrograph of an LED array integrated with a CMOS control chip. b) and $c$ ) Two examples of patterns belonging to the $16 \times 16$ Hadamard basis where white indicates the LED being on and black corresponds to the LED being off.

in the array switched on, thus providing a constant illumination level. The device records the intensity it receives from each pattern and can subsequently determine its position. Since the pattern sequence as well as an embedded clock signal for synchronization can be implemented in the transmission protocol, no uplink from the device to the ceiling light is required.

The scheme can be supplemented by technologies currently being developed for Li-Fi. For example, a faster modulation on top of the pattern sequence can enhance robustness to ambient light and compatibility with dimming. The knowledge of the device position can be exploited to provide individual data streams to devices in a multi-user Li-Fi environment.

\section{IMPLEMENTATION AND EARLY RESULTS}

We use a $16 \times 16$ array of $100 \times 100 \mu \mathrm{m}^{2}$ LED pixels emitting at $450 \mathrm{~nm}$. The LED array was bump-bonded to 

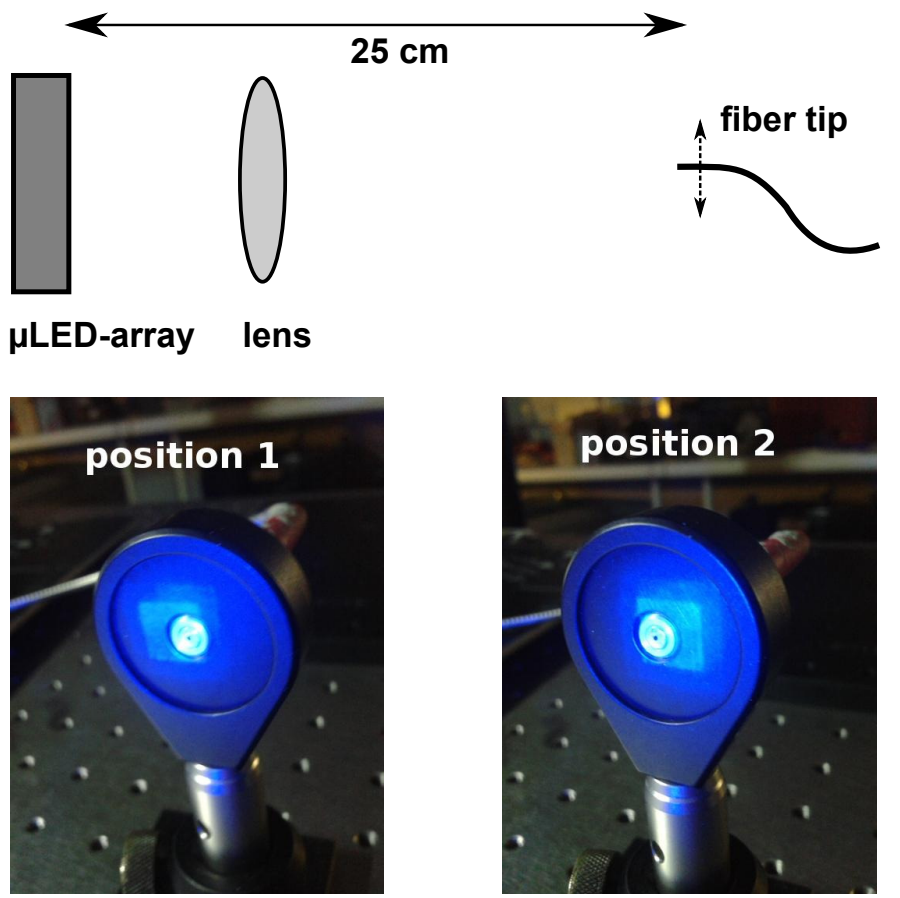

Fig. 3. Schematic of the initial experiment. The photographs show the optical fibre at two different positions. Note that the projected image of the microLED array can be seen on the fibre holder in these photographs.

a complementary metal oxide semiconductor (CMOS) driver chip enabling fully individual control of the pixels in a compact format [3], [4]. Figure 2a) shows such a CMOSintegrated LED array. A run through the full $16 \times 16$ Hadamard basis takes 2 minutes limited by the computer interface currently in use and also by the integration time required by the spectrometer that was used as a detector in this first demonstration. Note that the individual pixels in these devices respond with a full on-off modulation bandwidth of up to $110 \mathrm{MHz}$ [4]. Data rates in excess of $1 \mathrm{~Gb} / \mathrm{s}$ from a single micro-LED are possible with suitable encoding schemes [5]. A lens with $35 \mathrm{~mm}$ focal length was used to image the array at a distance of $25 \mathrm{~cm}$. The tip of an optical fiber $(50 \mu \mathrm{m}$ core diameter) coupled to a CCD spectrometer was placed in the imaging plane.

The configuration is shown in figure 3. During the test, the fiber was moved from position 1 to position 2 , which were $5 \mathrm{~mm}$ apart. Both raster-scanning and display of the Hadamard basis sequence were used to identify the position of the fiber tip. The results are shown in figure 4 . Using both methods, the position of the fiber can accurately be determined. Particular challenges that were not dealt with in this experiment are an electrical crosstalk that is particularly severe when several pixels are switched on at once and significant inhomogenity of the pixels. Despite these limitations, the positioning works well, illustrating that the proposed scheme is robust and is poised to take advantage of improvements in device performance.
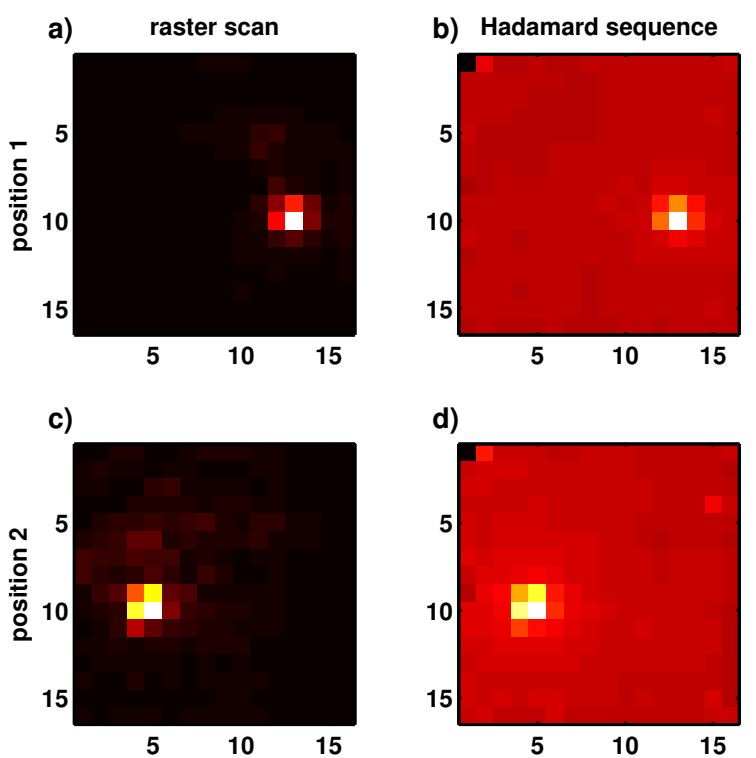

Fig. 4. Location signal for the fiber tip in the two positions shown in figure 3 , $a$ ) and $b$ ) position 1 and $c$ ) and $d$ ) position 2 . The signal was obtained by $a)$ and $c$ ) raster scanning and $b$ ) and $d$ ) reconstructed from the displayed Hadamard basis set.

\section{CONCLUSION AND OUTLOOK}

The basic concept of self-orientation within a room based on structured illumination by an arrayed ceiling lamp has been demonstrated. By developing systems that harness the $\mathrm{MHz}$ update rates of GaN micro-LED arrays, realtime autonomous navigation of an agent through an illuminated scence can be envisaged. Upcoming challenges are the development of more practical systems as well as combination with closely related technologies, in particular Li-Fi.

\section{ACKNOWLEDGMENT}

The authors thank the Engineering and Physical Sciences Research Council for funding under the grant EP/M01326X/1, QuantIC.

\section{REFERENCES}

[1] H. Burchardt et al., "VLC: Beyond Point-to-Point Communication," IEEE Commun. Mag., vol. 52, no. 7, pp. 98-105, 2014.

[2] N. Radwell et al., "Single-pixel infrared and visible microscope," Optica, vol. 1, no. 5, 2014

[3] J. J. D. McKendry et al., "Individually Addressable AlInGaN Micro-LED Arrays With CMOS Control and Subnanosecond Output Pulses," IEEE Photon. Technol. Lett., vol. 21, no. 12, pp. 811-813, 2009.

[4] - "Visible-Light Communications Using a CMOS-Controlled MicroLight-Emitting-Diode Array," J. Lightwave Technol., vol. 30, no. 1, pp. 61-67, 2012.

[5] D. Tsonev et al., "A 3-Gb/s Single-LED OFDM-Based Wireless VLC Link Using a Gallium Nitride $\mu$ LED," IEEE Photon. Technol. Lett., vol. 26 , no. 7 , pp. $637-640,2014$ 